\title{
Linking phytoplankton pigment composition and optical properties: a framework for developing remote-sensing metrics for monitoring cyanobacteria
}

Gabriel Hmimina ${ }^{\mathrm{a}}$, Florence D. Hulot ${ }^{\mathrm{a}}$, Jean François Humbert ${ }^{\mathrm{b}}$, Catherine Quiblier ${ }^{\mathrm{cd}}$, Kevin Tambosco $^{\mathrm{b}}$, Bruno J. Lemaire ${ }^{\mathrm{e}}$, Brigitte Vinçon-Leite ${ }^{\mathrm{e}}$, Louise Audebert ${ }^{\mathrm{a}}$, Kamel Soudani ${ }^{\mathrm{a}}$

${ }^{\text {a }}$ Ecologie Systematique Evolution, University of Paris-Sud, CNRS, AgroParisTech, Université Paris-Saclay, F-91400 Orsay, France

${ }^{\mathrm{b}}$ iEES Paris-INRA, Sorbonne Université, 75005 Paris, France

${ }^{c}$ Museum National d'Histoire Naturelle, UMR 7245 MNHN-CNRS, 75231 Paris, France

${ }^{\mathrm{d}}$ Université Paris Diderot, 75013 Paris, France

${ }^{\mathrm{e}}$ LEESU, Ecole des Ponts ParisTech, AgroParisTech, UPEC, UPE, Marne-la-Vallée, France

Corresponding author: Kamel Soudani: kamel.soudani@u-psud.fr

This is the post-print version of the article in Water Research, 2019, 148, pp.504 - 514. DOI: $10.1016 /$ j.watres.2018.09.055

Highlights :

- We developed a framework for the measurement of phytoplankton reflectance.

- $\quad$ It is used to validate remote-sensed cyanobacteria pigment concentrations.

- $\quad$ A systematic test of all possible 2-band and 3-band optical indices was performed.

- 2-band indices suffer from non-specificity issues.

- 3-band normalized difference indices yielded strong specific relationships.

\begin{abstract}
This study has been performed in the framework of a research program aiming to develop a low-cost aerial sensor for the monitoring of cyanobacteria in freshwater ecosystems that could be used for early detection. Several empirical and mechanistic remote-sensing tools have been already developed and tested at large scales and have proven useful in monitoring cyanobacterial blooms. However, the effectiveness of these tools for early detection is hard to assess because such work requires the detection of low concentrations of characteristic pigments amid complex ecosystems exhibiting several confounding factors (turbidity, blooms of other species, etc.). We developed a framework for performing high-throughput measurements of the absorbance and reflectance of small volumes $(\sim=20 \mathrm{~mL})$ of controlled mixtures of phytoplankton species and studied the potential of this framework to validate remote-sensing proxies of cyanobacteria concentration. The absorption and reflectance spectra of single and multiple cultures carried a specific signal that allowed for the quantitative analysis of culture mixes. This specific signal was shown to be related to known pigment absorbance spectra. The concentrations of chlorophyll- $a$ and $-b$, phycocyanin and phycoerythrin could be obtained from direct absorbance measurements and were correlated with the concentration obtained after pigment extraction $\left(R^{2} \geq 0.96\right.$ for all pigments). A systematic test of every possible two-band and three-band normalized difference between optical indices was then performed, and the coincidental correlation with chlorophyll- $b$ (absent in cyanobacteria) was used as an indicator of non-specificity. Two-band indices were shown to suffer from non-specificity issues and could not yield strong and specific relationships with phycocyanin or phycoerythrin (maximum $\mathrm{R}^{2}<0.5$ ). On the other hand, the three-band modified normalized difference indices yielded strong specific relationships $\left(\mathrm{R}^{2}>0.8\right)$.
\end{abstract}




\section{Introduction}

Inland waters often exhibit blooms of cyanobacteria due to eutrophication and, to a lesser extent, to climatic changes (Jöhnk et al. 2008; Pearl and Huisman 2008; Markensten et al. 2010). These blooms are responsible for ecosystem functioning disruptions, perturbations in recreational activities and/or disruptions in the production of drinking water, primarily because of the possible presence of toxic metabolites produced by certain cyanobacteria. The adverse effects of these cyanotoxins on human and animal health have been described (e.g., Kuiper-Goodman et al. 1999, Carmichael et al. 2001, Briand et al. 2003, Codd et al. 2005). Reducing the exposition of human populations to cyanotoxins requires an early detection and water body closure which can only be achieved through frequent monitoring. Current methods consist of regularly timed field sample collections in one or several points considered to be representative of the ecosystem or of the area of interest (bathing area, intake of water plant), followed by laboratory analysis identification and enumeration of phytoplankton. This approach is time and money consuming and sometimes poorly effective due to the complex spatiotemporal dynamics of cyanobacterial blooms (Porat et al. 2001, Welker et al. 2003, Cuypers et al. 2011; Pobel et al. 2011).

Although remote sensing may be an adequate tool for accessing temporal and spatial variations in cyanobacterial blooms, most previous efforts in this field have focused on estimating chlorophyll- $a$ as a proxy of global phytoplanktonic biomass in water (Mittenzwey et al., 1992). Few studies have approached the issue of measuring phycocyanin, a cyanobacterial-characteristic accessory pigment whose concentration is generally much lower than chlorophyll- $a$, thus making it harder to track (Li et al., 2015). The methods, which are based on known optical properties of phycocyanin, mostly fall into two categories. First, semiempirical biophysical models have been designed to derive pigment concentrations from variations in reflectance spectra (Dekker et al., 1991; Simis et al., 2005). These tools, which are based on radiative transfer models, have the advantage of providing explicit mechanistically derived estimations of pigment concentration based on reflectance measurements (in situ, airborne and satellite measurements). But the drawback of these approaches is that they require extensive calibration, intense computations and highresolution spectral measurements. Moreover, the optical properties of phycocyanin measured in vitro do not directly translate to reflectance variability in a straightforward way, and many environmental factors may hamper the determination of its concentration. Second, empirical statistical models have been derived from relationships between optical measurements and laboratory measured pigment concentrations (Dekker, 1993; Dekker et al., 1991; Schalles and Yacobi, 2000; Simis et al., 2005). While these methods, which are commonly used today, have been shown to perform well, they suffer from a reliance on statistical calibrations, which are sensor specific and in certain cases lake specific. Moreover, most of the optical sensors based on these approaches are active sensors, which are expensive and perform badly in turbid waters.

Consequently, the most cost-efficient and easiest method of tracking the development of cyanobacterial blooms at relevant temporal and spatial resolutions would be a technique that relies on passive sensors able to extract relevant optical information from a limited number of spectral bands. This optical information could then be used as a proxy for pigment concentrations using empirical relationships or biophysical models. However, the definition of a limited number of spectral bands of interest is challenging due to the multitude of factors potentially affecting spectral properties in aquatic ecosystems. The determination of wavelength bands of interest must be made based on an extensive dataset encompassing different species over a wide range of concentrations and mixtures. 
To this end, we implemented a comprehensive approach to identify the most appropriate wavelength bands for the determination of pigment concentrations of different species of cyanobacteria, chlorophytes, cryptophytes and diatoms under experimental conditions of mono- and mixed cultures. This study is the first step toward the development of a new reflectance-based sensor for estimating concentrations of cyanobacteria in inland waters. The general methodology of this study was (i) to design and validate a measurement platform and methods which provide reliable measurements of pigment concentrations; (ii) to assess the ability of reflectance measurements to quantitatively detect different species of phytoplankton, including cyanobacteria, in turbid samples and to identify the most relevant wavelength range for quantitative detection; and (iii) to test a full range of simple optical indices while taking potential confounding effects into account.

\section{Materials and Methods}

\subsection{Phytoplankton cultures and absorbance measurements}

A set of fourteen species belonging to various phytoplankton groups was selected for the absorbance and reflectance measurements. This set comprises three chlorophytes, one cryptophyte, six cyanobacteria and four diatom species (see Table 1 for the detailed list). In addition, one of the most common benthic cyanobacteria proliferating in lakes or rivers (Phormidium favosum) was added to this set (Quiblier et al., 2013).

Table 1: Species and strains used for the measurements and their corresponding culture conditions

\begin{tabular}{lllll}
\hline Type & Species & Strains & $\begin{array}{l}\text { Culture } \\
\text { medium }\end{array}$ & $\begin{array}{l}\text { Temperature } \\
\left({ }^{\circ} \mathbf{C}\right)\end{array}$ \\
\hline Chlorophytes & Chlorella vulgaris & CCAP 211/11b & BB & 20 \\
& Pediastrum boryanum & ALCP 56a & BB & 20 \\
& Scenedesmus obliquus & ALCP 349 & BB & 20 \\
\hline Cyanobacteria & Anabaena planctonica & PMC 624.10 & Z8 & 25 \\
& Aphanizomenon flos-aquae & PMC 97.07 & Z8 & 20 \\
& Dolichospermum planctonica & PMC 624.10 & Z8 & 20 \\
& Microcystis aeruginosa & PCC 78.20 & Z8 & 18 \\
& Microcystis sp. & PMC 816.12 & Z8 & 25 \\
& Phormidium favosum & PMC 240.04 & Z8 & 20 \\
& Planktothrix agardhii & PMC 75.02 & Z8 & 20 \\
\hline \multirow{2}{*}{ Diatoms } & Asterionella formosa & CCAP 1005/9 & DM & 18 \\
& Aulacoseira granulata & CCAP 1002/1 & DM & 18 \\
& Cyclotella sp & CCAP 1070/4 & DM & 18 \\
& Stephanodiscus hantzschii & CCAP 1079/4 & DM & 18 \\
\hline Cryptophytes & Cryptomonas pyrenoidifera & CCAP 979/66 & DM & 18 \\
\hline
\end{tabular}

The stock cultures of each species were cultivated in $250 \mathrm{~mL}$ Erlenmeyer flasks in incubation chambers at a constant temperature with an $18 \mathrm{~h}$ light and $8 \mathrm{~h}$ dark cycle (110 $\mu$ mol. $\left.\mathrm{m}^{-2} \cdot \mathrm{s}^{-1}\right)$ supplied by white LED panels. Three different culture media were used: BB 
(Bold's Basal Medium, Nichols and Bold, 1965), Z8 (Skulberg and Skulberg, 1985) and DM (Diatom Medium; Beakes et al., 1988). Species culture conditions are detailed in Table 1.

For absorbance and pigment measurements, cultures were grown in two Erlenmeyer flasks of $500 \mathrm{~mL}$ under the same conditions as the stock cultures. The medium was inoculated with the species stock cultures. Twice a week, $20 \mathrm{~mL}$ of each flask was sampled under sterile conditions for absorbance measurements. The absorbance spectrum in the range of $400 \mathrm{~nm}$ to $800 \mathrm{~nm}$ was obtained using standard $1 \mathrm{~cm}$ cuvettes in a laboratory spectrophotometer (UVVIS; HP 8453, Agilent, CA, US).

When saturation was reached in the absorbance at $680 \mathrm{~nm}$, which indicates that a high concentration is reached, the content of one of the two flasks was filtered on a $1.2 \mu \mathrm{m}$ Whatman glass fiber filter $(\mathrm{GF} / \mathrm{C})$. One half of each filter was placed in $10 \mathrm{~mL}$ of methanol for 24 hours at $4^{\circ} \mathrm{C}$ for chlorophyll-a measurement. After centrifugation of the methanol solution, the chlorophyll concentrations were estimated using the protocol proposed by Talling and Diver (1963). The second half of each filter was used for the extraction of chlorophylls, carotenoids and phycobiliproteins according to the protocol described in Echenique et al. (2016) and the absorbance spectra of the pigment extract were recorded.

The content of the second flask was used to mix two cultures (Species1:Species2), as listed in Table 2. The following nine combinations of Species1:Species2 ratios (based on culture volume) were tested: 0:10 (0), 1:9 (0.1), 2.5:7.5 (0.25), 4:6 (0.4), 5:5 (0.5), 6:4 (0.6), 7.5:2.5 (0.75), 9:1 (0.9), and 10:0 (1). The absorbance and reflectance (see the Reflectance measurements section below) were then measured for each mixed culture.

Table 2: Species associations in mixed cultures (Species $1+$ each of Species 2)

\begin{tabular}{|c|c|}
\hline Species 1 & $\begin{array}{rr}\text { Species } 2 \\
\end{array}$ \\
\hline \multirow{7}{*}{ Microcystis sp. PMC 816.12} & Dolichospermum planctonica PMC 624.10 \\
\hline & Aphanizomenon flos-aquae PMC 97.07 \\
\hline & Asterionella formosa CCAP 1005/9 \\
\hline & Aulacoseira granulata CCAP 1002/1 \\
\hline & Chlorella vulgaris CCAP 211/11b \\
\hline & Pediastrum boryanum ALCP 56a \\
\hline & Stephanodiscus hantzschii CCAP 1079/4 \\
\hline \multirow{6}{*}{ Microcystis aeruginosa $\mathrm{PCC} 78.20$} & Aphanizomenon flos-aquae PMC 97.07 \\
\hline & Asterionella formosa CCAP 1005/9 \\
\hline & Aulacoseira granulata CCAP 1002/1 \\
\hline & Chlorella vulgaris CCAP 211/11b \\
\hline & Pediastrum boryanum ALCP 56a \\
\hline & Stephanodiscus hantzschii CCAP1079/4 \\
\hline \multirow{4}{*}{ Chlorella vulgaris CCAP 211/11b } & Asterionella formosa CCAP 1005/9 \\
\hline & Aulacoseira granulata CCAP 1002/1 \\
\hline & Planktothrix agardhii PMC 75.02 \\
\hline & Scenedesmus obliquus ALCP 349 \\
\hline \multirow{2}{*}{ Phormidium favosum PMC 240.04} & Aulacoseira granulata CCAP 1002/1 \\
\hline & Cyclotella sp CCAP 1070/4 \\
\hline
\end{tabular}

\subsection{Reflectance measurements}


Reflectance measurements were performed with an integrating sphere $(15.24 \mathrm{~cm}$ in diameter, model RTC-060-IG, Labsphere, North Sutton, NH, USA) placed on top of a 3Dprinted $20 \mathrm{~mL}$ black cuvette. This cuvette was specially designed for this study as a light trap: its bottom surface $\left(5.3 \mathrm{~cm}^{2}\right)$ is rough, paved with cone-shaped asperities to limit the reflectance of transmitted light by the cuvette walls and to maximize the sample surface to volume ratio. Five prototypes of the cuvette with a varying number of cones $(0,7,37,61)$ were printed with a black flexible PLA (polylactic acid) material using a CubeX 3D printer (3D Systems, Rock Hill, USA), and the reflectance of both the empty and water-filled cuvettes was measured to select the optimal configuration. The maximum cuvette reflectance was found to decrease with increasing number of cones, and the prototype with 61 cones (the maximum number which could be accurately printed) was selected.

The cuvette was placed under the sample port of the integrating sphere and illuminated at an $8^{\circ}$ incidence angle while samples were injected through the inlet. An optical fiber, with a diffuser (cosine corrector) screwed onto its end (Thorlabs, Inc.), was mounted in the sphere detector port and connected to a CCD spectrometer $(\mathrm{LT} 1,300-1100 \mathrm{~nm}, 0.33 \mathrm{~nm}$ resolution, ASEQ spectrometers, ASEQ Instruments). At the beginning of each measurement session, a reference panel covered with Spectralon, a fluoropolymer of highest diffuse reflectance (Labsphere, Inc.), was substituted for the cuvette to record a reference spectrum (incoming light). The total and diffuse reflectances (by exclusion of the specular reflectance, using a port opposed to the light source port) were measured for each sample. After each measurement, the cuvette was rinsed with the culture medium used for the following sample until no variation in the reflectance spectrum could be seen. The cuvette outlet was designed as a selfactivating syphon to prevent sample spillage inside the integrative sphere.

\subsection{Data analyses}

\subsubsection{Analysis of monoculture absorbance and reflectance}

The spectra acquired on monocultures were compiled in a database and analyzed using MATLAB (MathWorks, Inc.). An overview of the dataset is given in Table 3. The dataset was smoothed with a normalized Gaussian of full width at half maximum (FWHM) of $10 \mathrm{~nm}$ in the spectral dimension to remove residual noise.

Table 3: Number of acquired spectra for each phytoplankton group

\begin{tabular}{llll}
\hline & Pigment absorbance & Culture absorbance & Culture reflectance \\
\hline Chlorophytes & 18 & 1098 & 81 \\
Cyanobacteria & 29 & 1917 & 153 \\
Diatoms & 0 & 1038 & 99 \\
\hline Total & 47 & 4053 & 180 \\
\hline
\end{tabular}

\subsubsection{Analysis of mixed culture absorbance and reflectance}

In mixed cultures (Table 2), absorbance and reflectance were measured for all the mixing ratios. First, a regression was performed on each spectrum of the mixed culture, using the monoculture spectra as explanatory variables. The aim was to test the ability of a simple linear model to extract the species ratio in the mixed culture, where the model captures both mixed culture absorbance and reflectance spectra as a linear combination of monoculture 
spectra. For both absorbance and reflectance, the slopes of the regression of all samples were pooled and compared to the known ratios.

Second, the known ratios were used to estimate mixed culture spectra using the following linear model (Eq. 1). This model was applied for the distinct purpose of estimating both the reflectance and the absorbance of the culture:

$$
R_{e}(\lambda)=\sum_{s} p_{s} \times R_{s}(\lambda) \quad(\text { Eq. } 1)
$$

where $R_{e}$ represents the estimated mixed culture reflectance (or absorbance) at the wavelength $\lambda, R_{s}$ represents the monoculture reflectance (or absorbance), and $p_{s}$ represents the known ratio of the species $s$ in the mixed culture.

For each sample, the reflectance (or absorbance) estimated with Eq .1 was compared to the measured reflectance (or measured absorbance), and the estimation error was calculated as the normalized root mean square error (nRMSE) for each sample. Because the RMSE depends on the magnitude of reflectance (or absorbance), it was normalized. The normalization is given by the division of the RMSE by the average reflectance (or absorbance) over the whole wavelength range (300-1100 nm) according to the following equation:

$$
n R M S E=\frac{\sqrt{\frac{\sum_{\lambda}\left(R_{m}(\lambda)-R_{e}(\lambda)\right)^{2}}{L}}}{\frac{\Sigma_{\lambda} R_{m}(\lambda)}{L}}
$$

where $L$ represents the number of wavelengths symbolized by $\lambda$ in Eq. 2 , and $R_{m}(\lambda)$ represents the measured reflectance (absorbance) of the sample at wavelength $\lambda . R_{e}(\lambda)$ is the estimated reflectance (absorbance) from Eq. 1.

Because RMSE is not homogeneous over the whole wavelength range, for each wavelength $\lambda$, the reflectance (absorbance) estimation error was also calculated over the whole dataset (all samples) as follows:

$$
n R M S E(\lambda)=\frac{\sqrt{\frac{\sum_{i}^{N}\left(R_{m}(\lambda)-R_{e}(\lambda)\right)^{2}}{N}}}{\frac{\sum_{i}^{N} R_{m}(\lambda)}{N}}
$$

where $N$ represents the total number of samples.

\subsubsection{Estimation of cell pigment contents}

A pigment concentration extraction model, derived from the one described in Hoepffner and Sathyendranath (1993), was fitted to the measured absorbance of the strains and to the absorbance of pigments after extraction. In this model, the total absorbance of the sample is the linear combination of the absorbance of pigments, colored dissolved organic matter and chlorophyll degradation products.

This model was fitted in a constrained two-step nonlinear optimization using the fmincon function (optimization toolbox, MATLAB, MathWorks) and can be written as follows:

$$
\begin{aligned}
& A_{i}(\lambda)=a_{\text {CDOM }_{i}}(440) \times e^{-S_{\text {CDOM }_{i}}(\lambda-440)} \\
& +a_{\text {tryp }_{i}}(440) \times e^{-s_{\text {tryp }_{i}}(\lambda-440)}+\sum_{p} a_{p}\left(\lambda+d_{p}\right) \times C_{p, i} \quad \text { (Eq.4) }
\end{aligned}
$$

where $A_{i}$ represents the absorbance of the sample at the wavelength $\lambda ; a_{C D O M_{i}}$ and $s_{C D O M_{i}}$ represent two sample-dependent parameters related to the colored dissolved organic matter absorbance; $a_{\text {tryp }}$ and $S_{\text {tryp }}$ represent two sample-dependent parameters related to 
chlorophyll- $a$ degradation residual absorbance; $a_{p}$ represents the absorbance spectrum of the pigment $p$ measured after extraction from the sample; $d_{p}$ represents the wavelength shift between in vitro and in vivo absorbance spectra of the pigment $p$, and $C_{p, i}$ represents the concentration of sample $i$ in pigment $p$.

First, for each value of $d_{p}$, the sample-dependent parameters were optimized to minimize the observed versus predicted absorbance RMSE for each sample, and the mean coefficient of determination $r^{2}$ was computed for each sample. Second, the $d_{p}$ parameters were optimized to maximize the computed mean coefficient of determination over the whole dataset. The model was fitted over the absorbance spectra of the pigment extracts and over the culture absorbance spectra to estimate the $C_{p, i}$ for chlorophyll- $a$, chlorophyll- $b$, phycocyanin and phycoerythrin of each sample. The distribution of the computed coefficient of determination was checked, as well as the similarity between results computed based on pigment absorbance spectra and absorbance measurements of mono- and mixed cultures.

\subsubsection{Analysis of reflectance-based optical indices}

The reflectance spectra measured (Section 2.2) were initially smoothed with a 10 nanometer FWHM Gaussian bandwidth, which was similar to the bandwidth usually used in commercially available optical interference filters, to derive estimations of single-band reflectance measurements. The smoothed spectra were sampled at $1 \mathrm{~nm}$ resolution and, then, were used to compute every possible two- and three-band spectral index between $400 \mathrm{~nm}$ and $800 \mathrm{~nm}$.

For the two-band-based spectral indices or normalized difference indices (NDi), we investigated the following form:

$$
N D i=\frac{R_{\text {band } 1}-R_{\text {band } 2}}{R_{\text {band } 1}+R_{\text {band } 2}} \quad(\text { Eq.5) }
$$

where $R_{\text {band } 1}$ represents the measured reflectance in the band of interest, and $R_{\text {band } 2}$ represents the estimated reflectance in a reference band.

Similarly, three-band-based spectral indices or modified normalized difference indices (mNDi) have the following form:

$$
m N D i=\frac{R_{\text {band } 1}-R_{\text {band } 2}}{R_{\text {band } 1}+R_{\text {band } 2}-2 \times R_{\text {band } 3}} \quad \text { (Eq.6) }
$$

The 125 spectra exhibiting the highest pigment content retrieval $\left(r^{2}\right)$ were selected, and the correlation between spectral indices and pigment concentrations was computed. Only cyanobacteria strains were used for the computation of correlations between reflectance and phycocyanin and phycoerythrin content. In the same way, the correlation between reflectance and chlorophyll- $a$ and chlorophyll- $b$ pigment content was calculated only on chlorophyte strains.

For the two types of spectral indices (Eq.5 \& 6) and the four pigments, the correlations were mapped as a function of wavelength bands. A composite red-green-blue (RGB) image was produced, which shows the correlation between spectral indices and phycocyanin (red image layer), chlorophyll- $a$ (green) and chlorophyll- $b$ (blue) as color layers to illustrate the relative correlation between the couples in bands of interest and each pigment concentration.

\section{Results}

\subsection{Absorbance and reflectance of mixed cultures}


First, linear regressions were used to estimate the species ratios in mixed cultures based on the absorbance and reflectance spectra estimated for mixed cultures as the predicted variables and the absorbance and reflectance spectra estimated for monocultures as the predictor variables (Eq.1). The slopes of these regressions were pooled and their median and confidence intervals for each mixed culture ratio are shown in Fig. 1.

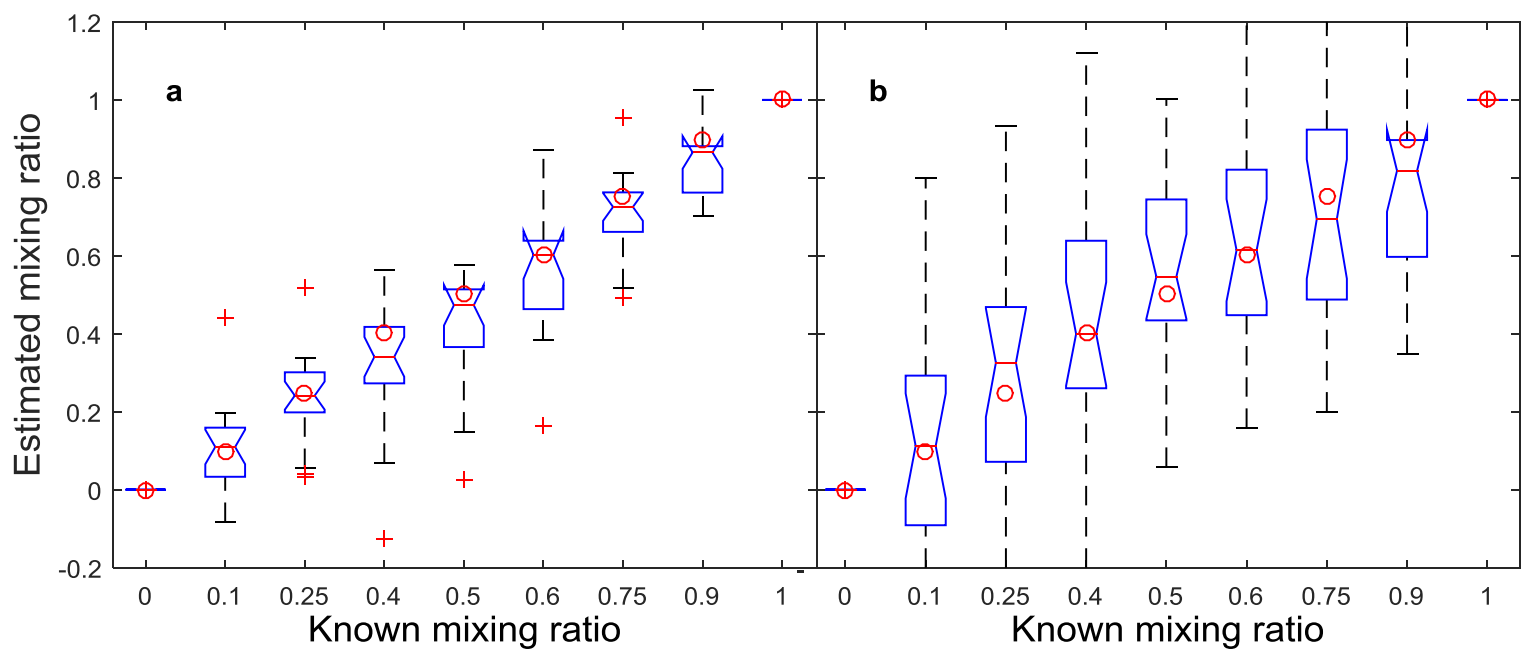

Fig. 1. Predicted ratios as a function of experimental ratios of Species 1 in the mixed cultures based on the absorbance (a) and reflectance (b). The red lines are the median of the predicted ratios, the notches indicate the $95 \%$ confidence interval around the median, and the red dots are the median ratios of the experimental mixed cultures. Red crosses are outliers.

The predicted and measured ratios for both reflectance- and absorbance-based estimations were consistent (Fig. 1). For the results based on absorbance measurements, the predicted concentrations of Species 1 in mixed cultures (Species 1 and Species 2, Table 2) correlated with the experimental concentrations as shown by the linear relationship found between the predicted and experimental ratios (Fig. 1a). A similarly good agreement between the predicted and measured concentrations was also found for Species 2 (data not shown). Moreover, the estimated ratios were included in the $95 \%$ confidence interval of the experimental ratios in all cases except two (Fig. 1a). For reflectance measurements, the predicted ratios exhibited a clear linear and positive trend with the experimental ratios, and the confidence intervals of the ratios displayed important overlaps (Fig. 1b) and were much larger than those found using absorbance (Fig. 1a and 1b). Nonetheless, the medians of the estimated ratios matched the medians of the experimental ratios and were contained within the $95 \%$ confidence intervals for all ratios.

The relative errors (Eq. 2) in reflectance estimations, which are based on the sums of individual species reflectance multiplied by the experimental concentration for each species over a large range of wavelengths $(475-750 \mathrm{~nm}$ ), were lower than 2.5\% (Fig. 2a). Most species displayed comparable relative errors except $P$. agardhii, $P$. boryanum and $D$. planctonica, which exhibited lower relative errors, and $S$. obliquus, which exhibited a higher relative error. These four species plus Cyclotella sp. also exhibited less variability in their relative errors.

The variation in the relative error in reflectance by wavelengths (Eq. 3) exhibits a clear pattern with three hollows in the 450-750 $\mathrm{nm}$ range (Fig. 2b). In order from the highest to the lowest relative error, the hollows occur at approximately $500 \mathrm{~nm}, 630 \mathrm{~nm}$, and $675 \mathrm{~nm}$ and correspond to carotenoid, phycocyanin and chlorophyll pigments, respectively. In addition, a 
peak can be seen at approximately $550 \mathrm{~nm}$, corresponding to an overlap between carotenoid, phycoerythrin and phycocyanin absorbance.
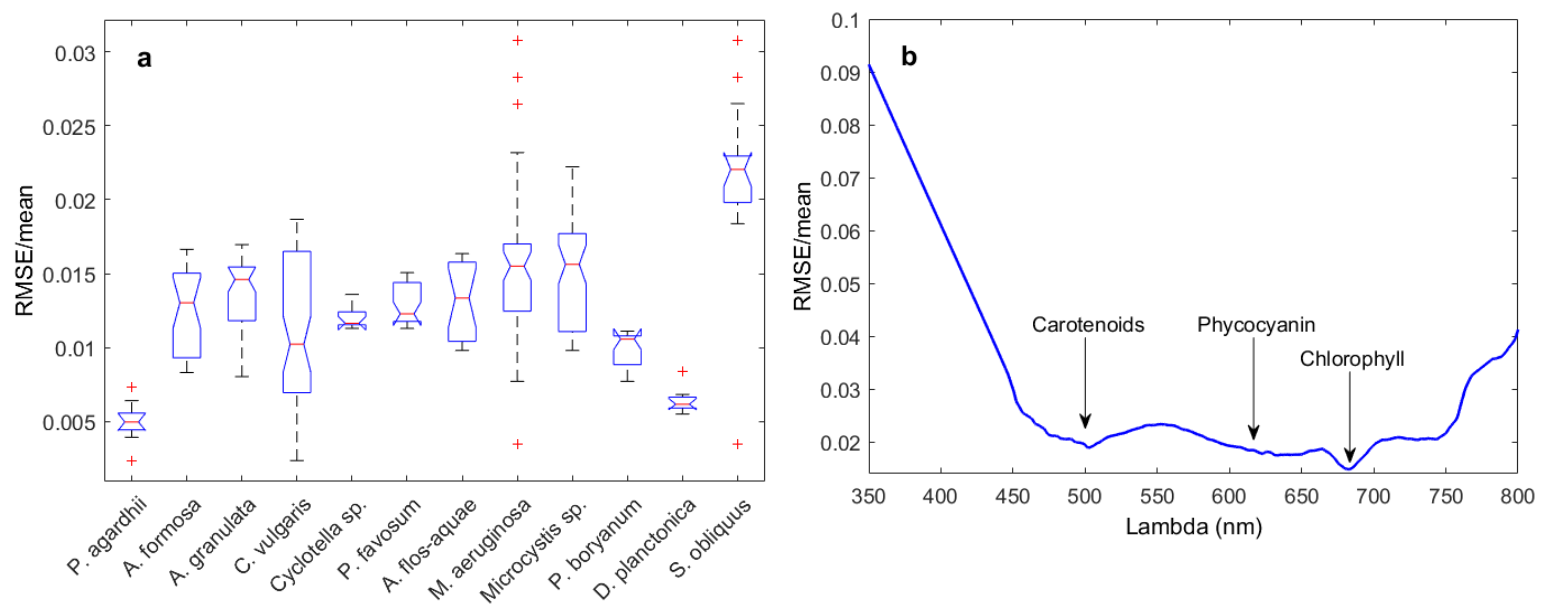

Fig. 2. Relative error in reflectance estimation based on the experimental mixing ratios for different species (a) and for different wavelengths in the visible spectrum (b). The boxes represent the sample median (red tick), the 95\% confidence interval around the median (notches) and the average $+/-$ standard deviation (box). Species (x-axis, left panel): $P$. agardhii, A. formosa, A. granulata, C. vulgaris, C. pyrenoidifera, Cyclotella sp., $P$. favosum, A. flos-aquae, M. aeruginosa PCC 78.20, Microcystis sp. PMC 816.12, P. boryanum, P. cinctum, D. planctonica, S. obliquus, S. hantzschii.

\subsection{Estimation of cell pigment content}

Supplemental Fig. 1 shows the comparison between the experimental absorbance spectra of the monocultures and the estimated absorbance spectra based on the absorbance of their pigments as calculated using Eq. 4. The model given in Eq. 4 accurately reproduces the pattern of absorbance in both extracted pigments and monocultures and adequately fits both the pigment-related features and the spectra baseline. The shift in pigment peak absorbance between the methanol solution containing the pigments and the in vivo spectrum can also be seen, particularly for the peak related to chlorophylls, which is shifted from $670 \mathrm{~nm}$ in methanol to $680 \mathrm{~nm}$ in vivo. This shift was also accurately reproduced by the model.

The distribution of the model's $R^{2}$ for all monocultures is shown in Supplemental Fig. 2. The distribution exhibits an exponential behavior in both cases, with a mode close to 1 . Outliers can be seen in the distribution's tail, below $R^{2}=0.95$ in extracted pigments, and below $R^{2}=0.6$ in monocultures.

Finally, the relationship between the estimated and the experimental pigment concentration in extracts and in monocultures (Fig. 3) showed a strong linear relationship for chlorophyll- $a\left(\mathrm{R}^{2}=0.9955\right)$, chlorophyll- $b\left(\mathrm{R}^{2}=0.9369\right)$ and phycocyanin $\left(\mathrm{R}^{2}=0.962\right)$, with the exception of a few outliers (excluded from regressions). For the phycoerythrin pigment, a highly significant relationship was found $\left(\mathrm{R}^{2}=0.956\right)$, but this relationship exhibited a lower sensitivity in the lowest range of the concentrations estimated after extraction (Fig.3). 

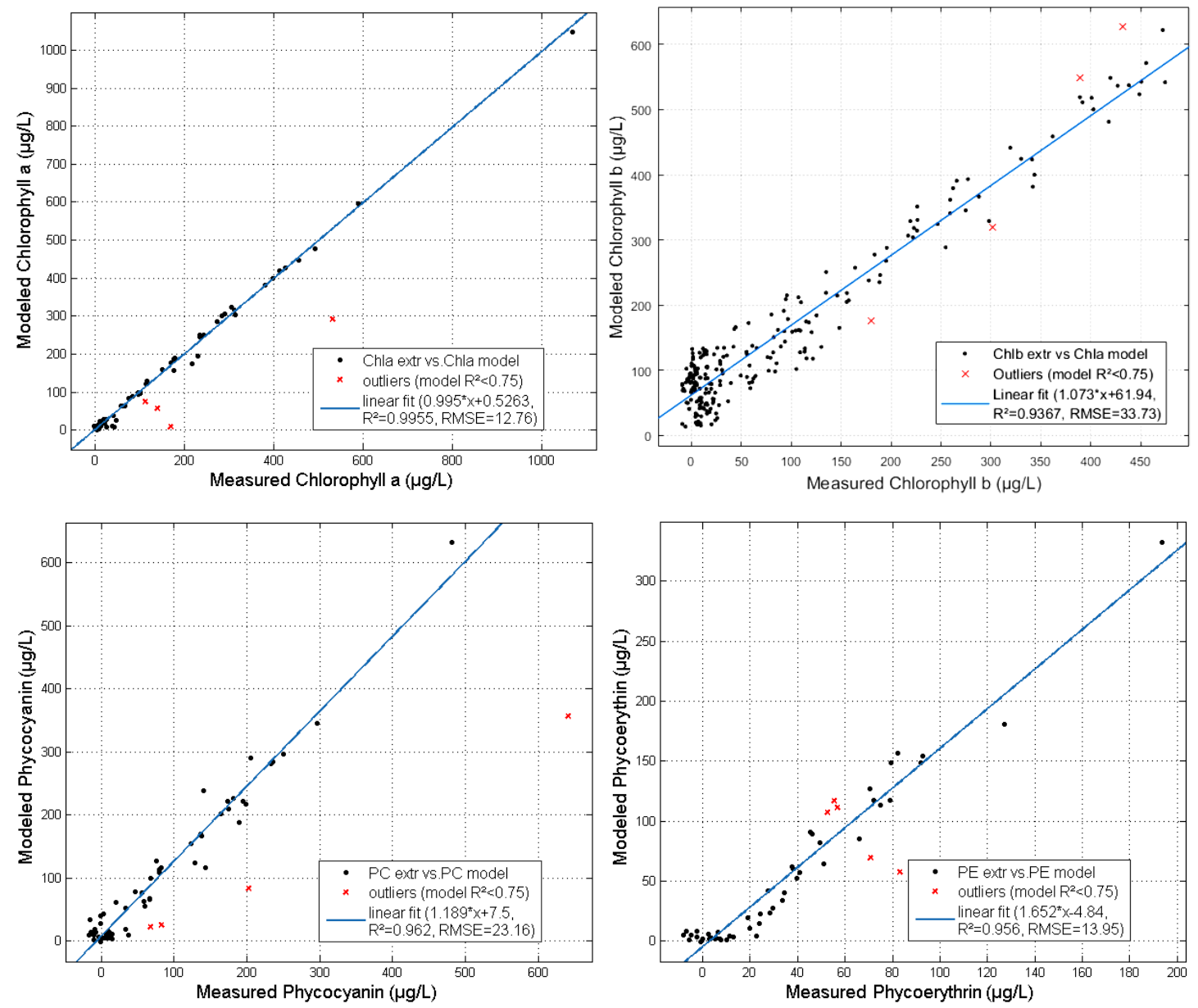

Fig. 3. Relationship between the estimated pigment (chlorophyll- $a$, chlorophyll- $b$, phycocyanin and phycoerythrin) concentrations measured after extraction and in vivo

\subsection{Analysis of reflectance-based optical indices}

\subsubsection{Two-band indices}

First, the reflectance of samples measured using the integrative sphere was used to calculate all possible NDi indices using Eq. 5 to simulate the reflectance-based prediction and to estimate pigments with a two-band sensor configuration. The results are shown in Fig. 4 for chlorophyll- $a$, chlorophyll- $b$, phycoerythrin and phycocyanin. 

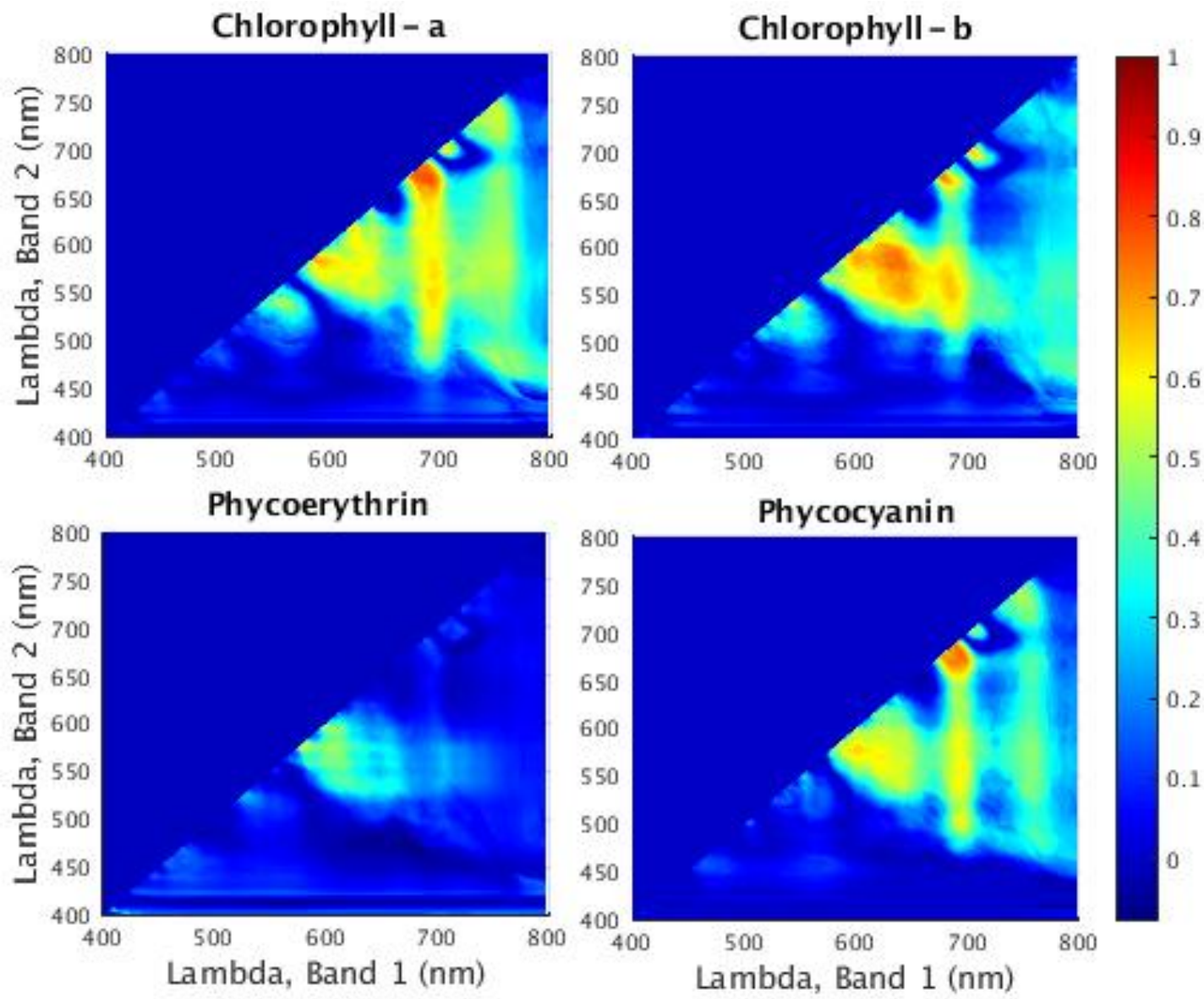

Fig. 4. Correlation map between the pigment content in the sample solutions and the NDi for all combinations of wavelengths in the visible spectrum. The scale bar on the right represents $\mathrm{R}^{2}$ values.

The map of correlations obtained for all pigments was then superimposed to provide a composite image showing the dependence between the spectral indices and the four pigments simultaneously (Fig. 5). A spot of deep green can be seen at approximately $550 \mathrm{~nm}$, indicating a high correlation of the NDi with chlorophyll- $a$ (color composition above the diagonal) and chlorophyll- $b$ (color composition below the diagonal) and a low correlation with phycocyanin and phycoerythrin. This result underlines the high and similar sensitivity of wavelengths of approximately $550 \mathrm{~nm}$ to chlorophyll-a and chlorophyll-b pigments. The deep yellow (green and red) large band centered at approximately $680 \mathrm{~nm}$, which was observed previously, indicates a similar correlation with both chlorophylls and phycocyanin. In both the top and bottom portions of the figure, a purple spot (red and blue) can be seen primarily in the vicinity of 625 and $450 \mathrm{~nm}$, indicating a high correlation with both phycocyanin and phycoerythrin. Finally, the large band, which can be seen between $700 \mathrm{~nm}$ and $775 \mathrm{~nm}$, in yellow in the top portion of the figure and mostly in red in the bottom portion indicates a strong correlation with both chlorophyll- $a$ and phycocyanin but a low correlation with chlorophyll- $b$. 


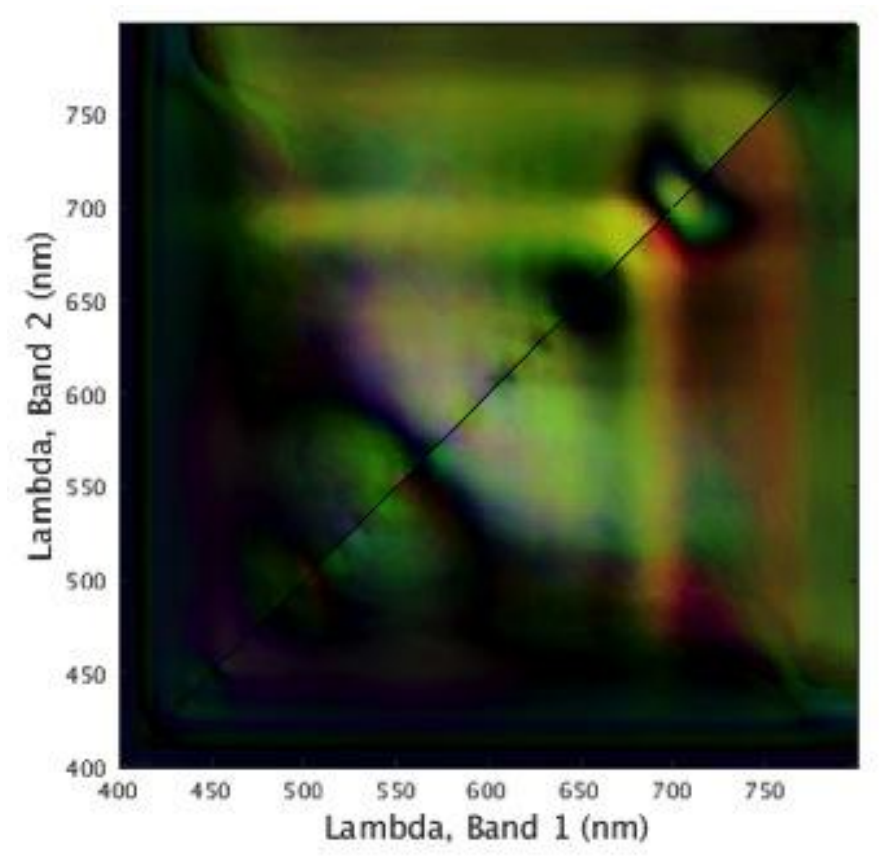

Fig. 5. RGB composite image showing the combined correlations between pigment concentration and the computed NDi by wavelength. The diagonal (black line) separates two different images with different color compositions: In the top left: red=phycocyanin, green=chlorophyll-a, blue=phycoerythrin. In the bottom right: red=phycocyanin, green=chlorophyll-b, blue=phycoerythrin. The two-color compositions should be read independently. See text for explanations of other colors.

\subsubsection{Three-band indices}

The relationships between the mNDi (Eq.6) and pigment concentrations are illustrated in Fig. 6. The third band (or second reference band), which was selected as the best performing, varies in a discrete manner, exhibiting large homogeneous patterns for the four pigments. The correlation between the $\mathrm{mNDi}$ and pigment concentrations exhibited more variability and clearer, more contrasted patterns than the NDi. The correlation patterns between chlorophyll$a$ and $-b$ were comparable, except for an overall lower correlation for chlorophyll- $b$. The correlation patterns for phycocyanin and phycoerythrin shared certain similarities, but the latter showed an overall lower correlation and did not exhibit the patches of high correlation for band 1 at approximately $710 \mathrm{~nm}$, which can be seen for phycocyanin. These patterns exhibited a significant overlap with the pattern observed for chlorophyll- $a$ and $-b$.

The maximum correlations for each pigment were observed using the following band combinations:

- Chlorophyll-a: $\{728,696,684\}: R^{2}=0.979$

- Chlorophyll-b: $\{736,688,682\}: R^{2}=0.768$

- Phycocyanin: $\{610,515,602\}: R^{2}=0.949$

- Phycoerythrin: $\{710,504,540\}: R^{2}=0.719$ 

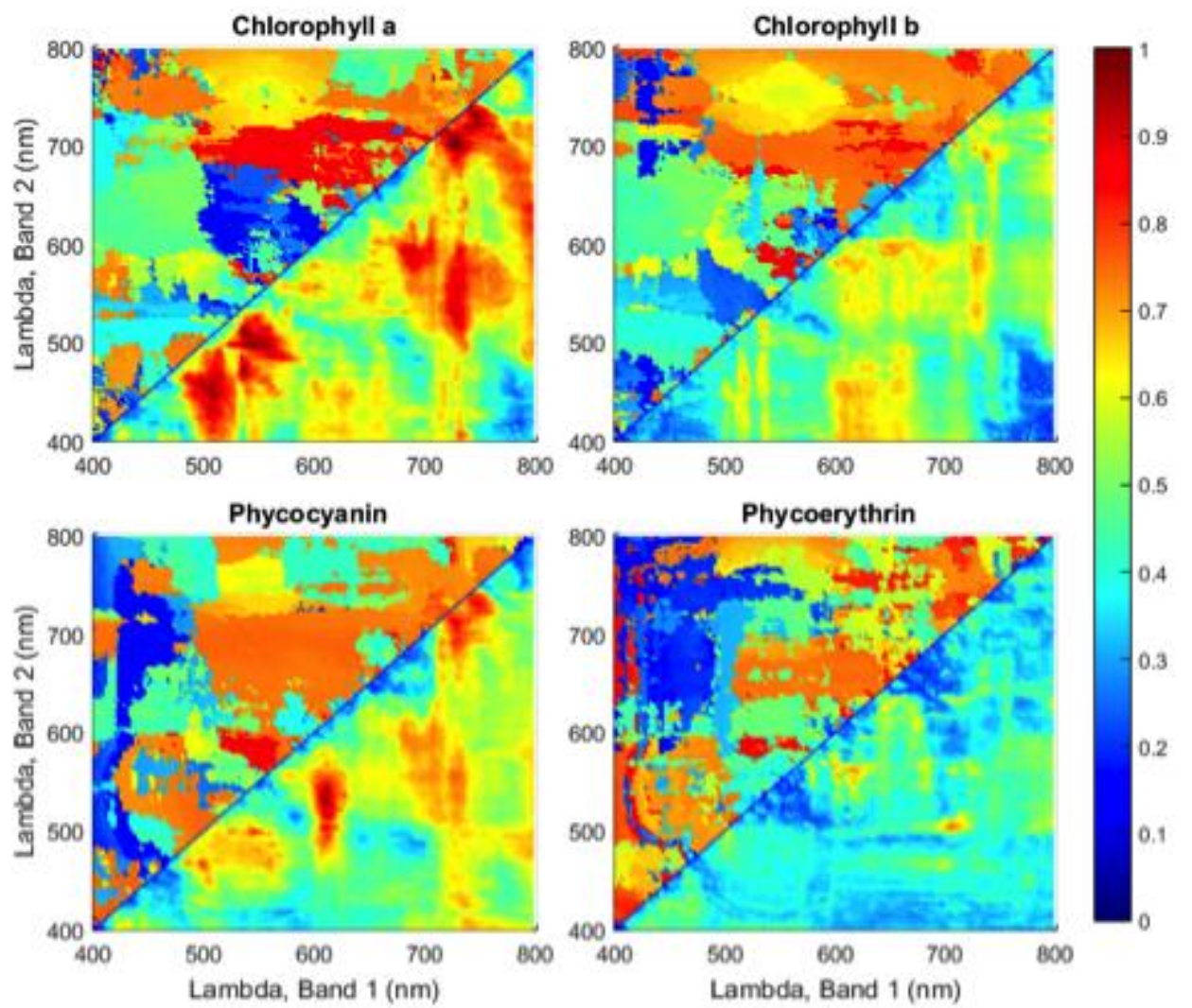

Fig. 6. Below the diagonal and left color scale: Correlation map between pigment content and the mNDi by the wavelengths of bands 1 and 2 . The scale bar on the right represents $\mathrm{R}^{2}$ values. Above the diagonal and the right most color scale: best performing third band wavelength (nm).

Because phycoerythrin clearly exhibited different patterns and lower correlations than the three other pigments, it has been excluded from the following analysis.

The combination of the correlation maps obtained for chlorophyll-a and -b and phycocyanin is shown in Fig. 7. While the overall pattern of the optimum third band shown in the top left parts of the graphs was comparable for chlorophyll- $a$ and $-b$ and phycocyanin (Fig. 6), a high variability of colors was seen after superposition, meaning that the optimum third band depends on the pigment being considered. The color-saturated areas indicate the range of wavelengths where the choice of the third band yielded a very different wavelength for each pigment. The dark, gray and clear areas remaining indicate the range of wavelengths where similar wavelengths were selected as the third band.

On the map, in the area below the diagonal, the combined correlation patterns exhibit low tone variability but are bright overall, indicating a high correlation between the concentration of the three pigments. Variations in color indicate differences in relative correlation with different pigments. Purple to red patches (correlation with chlorophyll- $b$ and phycocyanin) can be seen. An orange to red patch (correlation with phycocyanin) can be seen as well as a green patch (correlation with chlorophyll-a). Small light-blue patches (correlation with chlorophyll- $a$ and $b$ ) appear sporadically. 


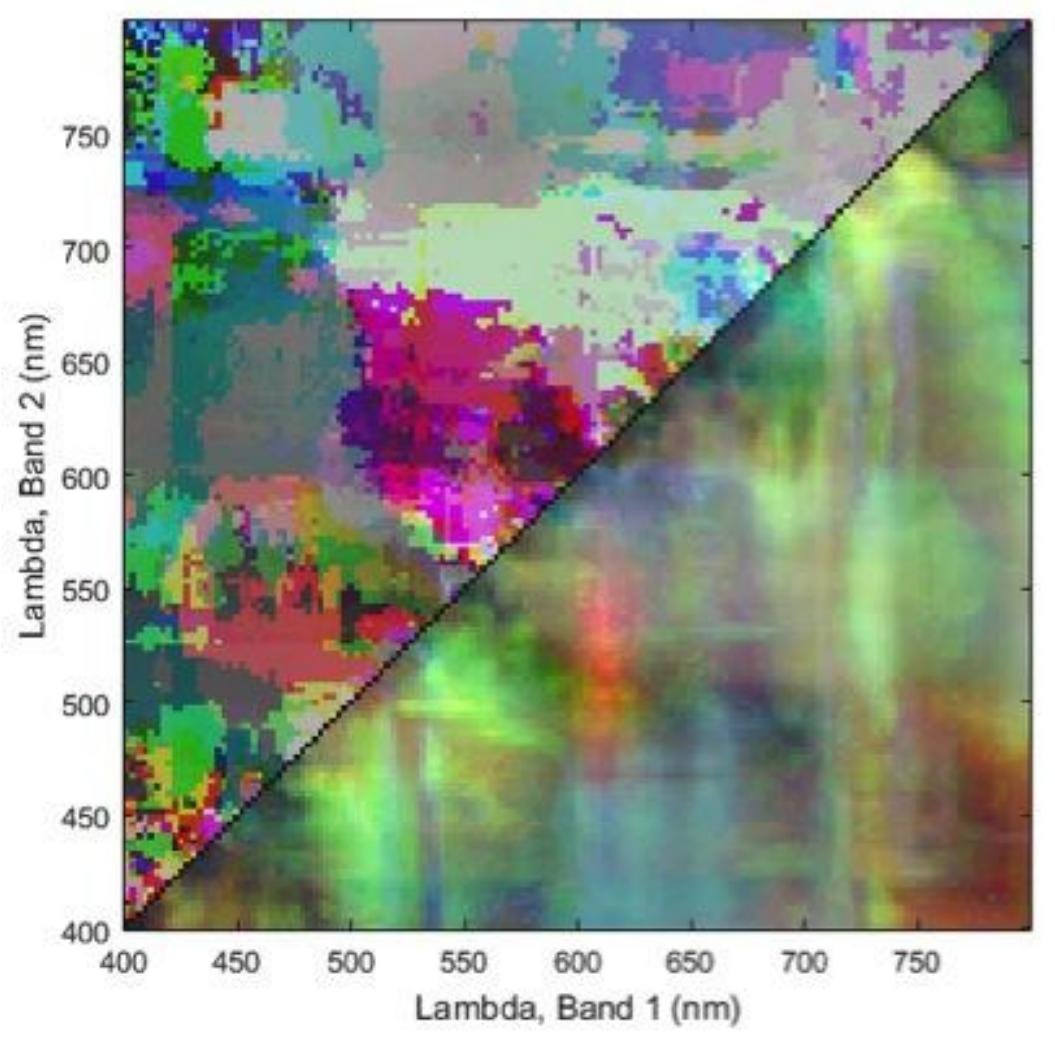

Fig. 7. Composite image showing combined correlations as a function of band wavelength (below the diagonal). Color intensity reveals the correlation between the three-band mNDi and chlorophyll-a concentration (green), phycocyanin concentration (red), and chlorophyll-b concentration (blue). Above the diagonal: best performing third reference band wavelength (nm).

\section{Discussion and conclusion}

We designed a new efficient device coupling an integrative sphere and a light-trapping cuvette, which allowed us to obtain accurate reflectance measurements. Indeed, when measuring the reflectance of liquid solutions, one of the most important issues is the relative contribution of the recipient to the resulting reflectance spectrum. For small liquid volumes, this contribution is mainly due to the back reflection of light transmitted through the solution by the recipient walls, which results in a mix of the reflectance of the liquid and the reflectance of the recipient.

The measurement platform developed in this study, which was based on a customdesigned cuvette and an integrative sphere, was designed to reduce the artifacts caused by back reflection of transmitted light and to optimize both the solution surface to volume ratio and the measurement sensitivity. The issues of back reflection and solution volume were solved with the use of light-trap geometry, which allowed the absorbance of most of the light transmitted through the solution. The sensitivity of the measurement is also optimized by using an integrative sphere, which collects most of the reflected light compared with the direct measurements above the solution.

The efficiency of this platform was assessed via the estimation of specific signatures of algae and cyanobacteria monocultures and the prediction of the reflectances of mixed cultures based on specific signatures. For both absorbance and reflectance, we accurately estimated the mixed culture composition based on the specific reflectance of each strain. The extraction of 
mixed culture reflectance based on monoculture reflectance was also accurate for all species and for each species. Using simple linear regression models, we were able to exclude any significant contribution of complex nonlinear phenomena such as geometric effects or back reflection of transmitted light.

This approach also permits the quantification of the contribution of each wavelength to the global error in the prediction of reflectance over all mixed cultures. Indeed, two major components are known to contribute to the reflectance spectra: (1) the effect of pigments, whose concentrations are known to constitute a highly specific signature at the class and species level (Wright and Jeffrey, 2006); and (2) the effect of turbidity, which varies for each culture, even within the same species. Few errors were found for wavelengths consistent with the absorption wavelengths of major specific pigments (chlorophyll- $a$, $-b$, phycoerythrin, phycocyanin, carotenoids), whereas a high number of errors were recorded for lower wavelengths known to be impacted mostly by the effects of turbidity (Mishra et al. 2013). This result indicates that the turbidity in each culture contributes only a small amount to its specific signature and that differences in pigment content are the best predictors of mixed culture absorbance and reflectance spectra.

Although the extraction of complex solution pigment content usually relies on first filtering the solutions and extracting the pigments to limit the confounding effects of turbidity, several extraction algorithms have been proposed. These algorithms are able to deconvolve the contribution of pigment content from the contribution of dissolved organic matter and other turbidity components (Ficek et al. 2004; Mishra et al. 2012, 2013, 2014). Using the same type of approach, which relies on simple linear regression models based on known spectra of pure pigments, we were able to accurately model both the absorbance of extracted pigments and that of pure cultures. The model accuracy for monocultures was comparable to extracted pigment in terms of absorbance prediction accuracy and consistency, which is illustrated by the similar $R^{2}$ values and distribution and by the estimated pigment content. The reported $R^{2}$ values for both extracted pigment and pure culture media are comparable to the $R^{2}$ reported in Moisan et al. (2011).

Moreover, the relationship between the estimated pigment concentration of extracted pigments and monocultures was highly linear and exhibited a high $R^{2}$ and a low error. Although previous studies have had comparable success in using the same kinds of methods to estimate pigment concentrations from absorption spectra of particulate matter or from pure samples (Bidigare et al. 1989; Hoepffner and Sathyendranath. 1993; Moisan et al. 2011; Chase et al. 2013), this represents, to our knowledge, the first approach successfully applied to cultures displaying varying ages and turbidity. The direct use of cultures is a noteworthy improvement over the classical use of extracted pigments because (1) it is significantly less time-consuming, as the lengthy steps of filtering the solution and extracting the pigments are avoided; (2) small volumes can be used, as there is no need to concentrate the solution nor any loss due to extraction; and (3) it is less expensive, as it does not require the use of consumables, such as solvents and filters. This step is key to building a large database of absorbance and reflectance spectra of known mixtures of phytoplankton species with varying concentrations, as the number of measurements needed to describe combinations increases quickly with the number of species and concentrations tested.

The resulting database of reflectance spectra and associated pigment concentrations in numerous mixed cultures was used to test the relationship between all possible two-band and three-band optical indices and the concentration of each pigment. The use of mixed cultures enabled us to test these correlations in samples exhibiting realistic confounding-mixture effects and to accurately and selectively estimate the concentration of each pigment. The correlation maps for chlorophyll- $a$ and $-b$ and phycocyanin and phycoerythrin show significant overlaps. These overlaps may be explained by two distinct phenomena. First, the 
concentration of the different pigments can covary due to co-occurrence. For instance, chlorophyll- $a$ and phycocyanin concentrations are expected to both increase when cyanobacterial biomass increases, which may yield coincidental relationships between the optical index and the pigment concentration. Second, the absorption spectra of several pigments exhibited strong overlaps. This second phenomenon means that certain optical indices are not pigment specific. The use of such nonspecific indices may lead to pigment concentration overestimation. For instance, the use of an optical index that correlates with both chlorophyll- $a$ and phycocyanin to track cyanobacterial blooms may grossly overestimate cyanobacterial growth by including the contribution of microalgae.

The use of controlled mixtures alleviates the first one by introducing changes in algae concentrations, which reduces the coincidental correlation between cyanobacteria-specific pigments and chlorophyll- $a$ concentrations. The use of mixes of cyanobacteria and algaes also enables us to use the specificity of chlorophyll- $b$ to rule out co-occurrence in certain cases. While a high correlation with chlorophyll- $a$, phycocyanin and phycoerythrin can be due to both co-occurrence and spectral overlap, a high correlation with chlorophyll- $b$, phycocyanin and phycoerythrin can be due to only a spectral overlap because cyanobacteria do not contain chlorophyll- $b$. This was illustrated in Fig. 7, where the yellow areas in both the top and bottom portions correspond to indices exhibiting a lack of specificity, responding to chlorophyll- $a$ and $-b$ and phycocyanin content. On the other hand, in the same figure, the yellow areas in the upper portion that correspond to red areas in the bottom portion may point to phycocyanin-specific optical indices. These areas may also represent a response to chlorophyll- $a$ content caused by sampling bias and co-occurrence. Overall, the areas exhibiting a high correlation between NDi and phycocyanin or phycoerythrin content correspond to areas with low specificity. While specific indices could be found for phycocyanin, they exhibit low $R^{2}$ (below 0.5). No specific index could be found for phycoerythrin. The use of NDi based on two spectral bands does not seem to provide both the high correlation and specificity needed for cyanobacterial quantitative tracking.

We have shown also that the mNDi correlation maps appear to have much more contrast than the NDi correlation maps. In particular, the correlation map for phycocyanin content exhibits a pattern of high correlation for wavelengths of approximately $610 \mathrm{~nm}$ and $575 \mathrm{~nm}$ that does not completely overlap with areas of high correlation with chlorophyll-b. Therefore, the corresponding mNDi shows high correlation and high specificity for phycocyanin. The highest correlation (yellow area in the bottom portion of Fig. 7) indicates a significant correlation with chlorophyll-a content, which results from the co-occurrence of this pigment rather than from a lack of selectivity. A high correlation can be seen for all pigments for indices involving near infrared bands (centered on $700 \mathrm{~nm}$ ), which may be explained by changes in scattering associated with cultures cell density (Kudela et al. 2015).

The use of a third spectral band provides a clear improvement in spectral indices specificity and may allow for the specific and quantitative tracking of cyanobacteria. The resulting wavelengths of interest are noticeably different than the ones used in most retrieval algorithms (Li et al. 2013, Mishra et al. 2013, Song et al. 2013, Yacobi et al. 2015). While the difference in methods makes a direct comparison hazardous, it raises questions regarding the ability of band-based methods using nonspecific wavelength to accurately deconvolve the effects of changes in turbidity, chlorophyll content and phycocyanin content. The bands used in a standard index-based framework seem to provide insufficient specificity, which indicates that the accuracy of these methods may rely entirely on the validity of their hypotheses.

The promising indices we obtained in this study may underperform in realistic situations because of external factors not considered in this study. For instance, sensitivity to geometric effects or changes in water color, transparency and scattering properties may alter the performance of the indices. Further work is, therefore, needed in natural conditions to check 
that the optical indices can be used in situ. Nevertheless, to date, most of the available remotesensing tools for tracking cyanobacterial blooms rely on the detection of chlorophyll content variability (Hu et al. 2010, 2012; Mishra and Mishra 2012).

Efforts have also focused on tracking the concentration variability in cyanobacteriaspecific pigments (Simis et al. 2005; Wynne et al. 2008; Ogashawara et al. 2013; Lyu et al. 2013; Mishra et al. 2013, 2014) either by using empirically derived statistical relationships and/or by fitting to mechanistic models, which relate reflectance to absorbance characteristics. Most of the recent efforts have focused on the second category, which is seen as a way to alleviate confounding factors. In particular, the variability in chlorophyll content, echoed by its reflectance, overlaps with the effects of changes in phycocyanin content (Yacobi et al. 2015). The main weakness of mechanistic models is that these modes rely on numerous hypotheses that are seldom tested before use. Additionally, these models are generally fitted to large-scale observations, mostly related to bloom events, and do not allow a direct estimation of confounding factor effects (Stumpf et al. 2016). During cyanobacterial blooms, phycocyanin concentration is strongly correlated to chlorophyll concentration, as cyanobacteria possess both pigments. This strong correlation may generate incidental correlations between the resulting metrics and phycocyanin content, which may lead to the overestimation of phycocyanin content, for instance, in the case of non-cyanobacterial blooms.

In conclusion, indices exhibiting sufficient correlation and specificity for phycocyanin were obtained in this study, indicating that standard optical indices based on 3 bands can be used to track cyanobacterial growth instead of methods relying on models based on strong hypotheses or full-spectrum analysis algorithms.

\section{Acknowledgments}

This work was supported by the ANR OSS-CYANO project - ANR-13-ECOT-0001, grant ANR-2013-Ecotechnologies \& EcoServices (ECO-TS) of the French Agence Nationale de la Recherche. This project has also benefited from technical and human resources provided by CEREEP-Ecotron IleDeFrance (CNRS/ENS, UMS 3194). The authors express their great thanks to all partners for the very enriching discussions over the life of the OSS-CYANO project. The authors would like to thank ESE (University of Paris-Sud/CNRS/AgroParisTech, UMR 8079) laboratory members and more especially Gwendal Latouche, Zoran Cerovic and Sophie Thibault for their expert technical advice regarding experimentation in Phytoplankton cultures and pigment quantification. 


\section{References}

Beakes, G.W., Canter, H.M., Jaworski, H.M., 1988. Zoospore ultrastructure of Zygorhizidium affluens and Z.planktonicum, two chytrids parasitizing the diatom Asteriunella formosa. Can. J. Bot. 66, 1054-1067.

Bidigare, R.R., Morrow, J.H., Kiefer, D.A., 1989. Derivative analysis of spectral absorption by photosynthetic pigments in the western Sargasso Sea. J. Mar. Res. 47, 323-341.

Briand, J.-F., Jacquet, S., Bernard, C., Humbert, J.-F., 2003. Health hazards for terrestrial vertebrates from toxic cyanobacteria in surface water ecosystems. Vet. Res. 34, 361-77.

Carmichael, W.W., Azevedo, S.M.F.O., An, J.S., Molica, R.J.R., Jochimsen, E.M., Lau, S., Rinehart, K.L., Shaw, G.R., Eaglesham, G.K., 2001. Human Fatalities from Cyanobacteria: Chemical and Biological Evidence for Cyanotoxins. Environ. Health Perspect., 109, 663-668.

Chase, A., Boss, E., Zaneveld, R., Bricaud, A., Claustre, H., Ras, J., Dall'Olmo, G., Westberry, T.K., 2013. Decomposition of in situ particulate absorption spectra. Methods Oceanogr 7, 110-124.

Codd, G., Lindsay, J., Young, F., Morrison, L., Metcalf, J., 2005. Harmful Cyanobacteria. In Harmful cyanobacteria, eds. J. Huisman, H.C.P. Matthijs and P.M. Visser, 1-23. Netherlands: Springer.1-23.

Cuypers, Y., Vinçon-Leite, B., Groleau, A., Tassin, B., Humbert, J.-F., 2011. Impact of internal waves on the spatial distribution of Planktothrix rubescens (cyanobacteria) in an alpine lake. ISME J., 5, 580-589.

Dekker, A. G., Peters, S.W.M. 1993. The use of the Thematic Mapper for the analysis of eutrophic lakes: A case study in the Netherlands. Int. J. Remote Sens., 14, 799-821.

Dekker, A.G., Malthus, T.J., Seyhan, E., 1991. Quantitative modelling of inland water quality for high-resolution MSS systems. IEEE Trans. Geosci. Remote S., 19, 89-95.

Echenique-Subiabre, I., Dalle, C., Duval, C., Heath, M. W., Couté, A., Wood, S. A., Humbert, J.-F., Quiblier, C. 2016. Application of a spectrofluorimetric tool (bbe BenthoTorch) for monitoring potentially toxic benthic cyanobacteria in rivers. Water Res., 101, 341-350.

Ficek, D., Kaczmarek, S., Stoń-Egiert, J., Woźniak, B., Majchrowski, R., Dera, J., 2004. Spectra of light absorption by phytoplankton pigments in the baltic; conclusions to be drawn from a gaussian analysis of empirical data. Oceanologia, 46, 533-555.

Hoepffner, N., Sathyendranath, S.,1993. Determination of the major groups of phytoplankton pigments from the absorption spectra of total particulate matter. J. Geophys. Res., 98, 22789.

Hu, C., Cannizzaro, J., Carder, K.L., Muller-Karger, F.E., Hardy, R., 2010. Remote detection of Trichodesmium blooms in optically complex coastal waters: Examples with MODIS full-spectral data. Remote Sens. Environ., 114, 2048-2058.

Hu, C., Lee, Z., Franz, B., 2012. Chlorophyll a algorithms for oligotrophic oceans: A novel approach based on three-band reflectance difference. J. Geophys. Res. Oceans, 117, C01011.

Jöhnk, K.D., Huisman, J., Sharples, J., Sommeijer, B., Visser, P. M., Stroom, J.M., 2008. Summer heatwaves promote blooms of harmful cyanobacteria. Glob. Change Biol., 14, 495-512.

Kudela R.M., Palacios S.L., Austerberry D.C., Accorsi E.K., Guild L.S., Torres-Perez J., 2015. Application of hyperspectral remote sensing to cyanobacterial blooms in inland waters, Remote Sens. Environ., 167, 196-205.

Kuiper-Goodman T., Falconer I.R., Fitzgerald J., 1999. Human Health Aspects. In: Chorus I., Bartram J., editors. Toxic Cyanobacteria in Water: A Guide to Their Public Health Consequences, Monitoring and Management. E \& FN Spon; London, UK, 113-153. 
Li, L., Li, L., Song, K., 2015. Remote sensing of freshwater cyanobacteria: An extended IOP Inversion Model of Inland Waters (IIMIW) for partitioning absorption coefficient and estimating phycocyanin. Remote Sens. Environ., 157, 9-23.

Li, L., Li, L., Song, K., Li, Y., Tedesco, L. P., Shi, K., Li, Z., 2013. An inversion model for deriving inherent optical properties of inland waters: Establishment, validation and application. Remote Sens. Environ., 135, 150-166.

Lyu, H., Wang, Q., Wu, C., Zhu, L., Yin, B., Li, Y., Huang, J., 2013. Retrieval of phycocyanin concentration from remote-sensing reflectance using a semi-analytic model in eutrophic lakes. Ecol. Inform., 18, 178-187.

Markensten, H., Moore, K., Pearson, I., 2010. Simulated lake phytoplankton composition shifts toward cyanobacteria dominance in future warmer climate. Ecol. Appl., 20, 752 767.

Mishra, S., Mishra, D.R., 2012. Remote sensing of environment normalized difference chlorophyll index: A novel model for remote estimation of chlorophyll- a concentration in turbid productive waters. Remote Sens. Environ., 117, 394-406.

Mishra, S., Mishra, D. R., Lee, Z.,2014. Bio-optical inversion in highly turbid and cyanobacteria-dominated waters. IEEE Trans. Geosci. Remote Sens., 52, 375-388.

Mishra, S., Mishra, D. R., Lee, Z., Tucker, C.S., 2013. Quantifying cyanobacterial phycocyanin concentration in turbid productive waters: A quasi-analytical approach. Remote Sens. Environ., 133, 141-151.

Mittenzwey, K. H., Ullrich, S., Gitelson, A. A., Kondratiev, K.Y., 1992. Determination of chlorophyll-a of inland waters on the basis of spectral reflectance. Limnol. Oceanogr., 37, 147-149.

Moisan, J. R., Tiffany, A., Moisan, H., Linkswiler, M.A., 2011. An inverse modeling approach to estimating phytoplankton pigment concentrations from phytoplankton absorption spectra. J. Geophys. Res. Oceans, 116, C006786

Nichols, H.W., Bold, H.C., 1965. Trichosarcina polymorpha Gen. et Sp. Nov. J. Phycol., 1, 34-38.

Ogashawara, I., Mishra, D.R., Mishra, S., Curtarelli, M.P., Stech, J. L. 2013. A performance review of reflectance based algorithms for predicting phycocyanin concentrations in inland waters. Remote Sens., 5, 4774-4798.

Pearl, H.W., Huisman, J., 2008. Blooms like it hot. Science, 320, 57-58.

Pobel, D., Robin, J. Humbert, J.-F., 2011. Influence of sampling strategies on the monitoring of cyanobacteria in shallow lakes: Lessons from a case study in France. Wat. Res., 45, 1005-1014.

Porat, R., Teltsch, B., Perelman, A., Dubinsky, Z., 2001. Diel Buoyancy Changes by the Cyanobacterium Aphanizomenon ovalisporum from a Shallow Reservoir. J. Plankt. Res., 23, 753-763.

Quiblier, C., Wood, S., Echenique-Subiabre, I., Heath, M., Villeneuve, A., Humbert, J.-F., 2013. A review of current knowledge on toxic benthic freshwater cyanobacteriaecology, toxin production and risk management. Wat. Res., 47, 5464-5479.

Schalles, J.F., Yacobi, Y.Z., 2000. Remote detection and seasonal patterns of phycocyanin, carotenoid and chlorophyll pigments in eutrophic waters. Ergeb. Limnol., 55, 153-168.

Simis, S.G.H., Peters, S.W. M., Gons, H.J., 2005. Remote sensing of the cyanobacterial pigment phycocyanin in turbid inland water. Limnol. Oceanogr., 50, 237-245.

Skulberg, O.M., Skulberg, R.. 1985. Planktic species of Oscillatoria (Cyanophyceae) from Norway. Arch. Hydrobiol. Suppl., 71, 157-174.

Song, K.S., Li, L., Li, Z.C., Tedesco, L., Hall, B., Shi, K., 2013. Remote detection of cyanobacteria through phytocyanin for water supply source using three-band model. Ecol. Inform., 15, 22-33. 
Stumpf, R.P., Davis, T. W., Wynne, T.T., Graham, J.L., Loftin, K.A., Johengen, T.H., Gossiaux, D., Palladino, D., Burtner, A., 2016. Challenges for mapping cyanotoxin patterns from remote sensing of cyanobacteria. Harmful Algae, 54, 160-173.

Talling, J. F. \& Driver, D. 1963. Some problems in the estimation of chlorophyll a in phytoplankton. In: Proc. Conf. on Primary Productivity Measurements, Marine and Freshwater, Univ. Hawaii, 1961. U.S. Atomic Energy Comm. TID-7633: 142-146.

Welker, M., Von Döhren, H., Täuscher, H., Steinberg, C.E.W., Erhard, M., 2003. Toxic Microcystis in shallow lake Müggelsee (Germany) - dynamics, distribution, diversity. Archiv Hydrobiol., 157, 227-248.

Wright, S.W., Jeffrey, S.W., 2006. Pigment markers for phytoplankton production. In J. K. Volkman (Ed.), Handbook of Environmental Chemistry, Volume 2: Reactions and Processes (Vol. 2 N, pp. 71-104). Berlin, Heidelberg: Springer Berlin Heidelberg.

Wynne, T.T., Stumpf, R.P., Tomlinson, M.C., Warner, R.A., Tester, P.A., Dyble, J., Fahnenstiel, G.L., 2008. Relating spectral shape to cyanobacterial blooms in the Laurentian Great Lakes. Int. J. Remote Sens., 29, 3665-3672.

Yacobi, Y.Z., Köhler, J., Leunert, F., Gitelson, A.A., 2015. Phycocyanin-specific absorption coefficient: Eliminating the effect of chlorophylls absorption. Limnol. Oceanogr. Met., $13,157-168$. 


\section{Supplemental Figures}
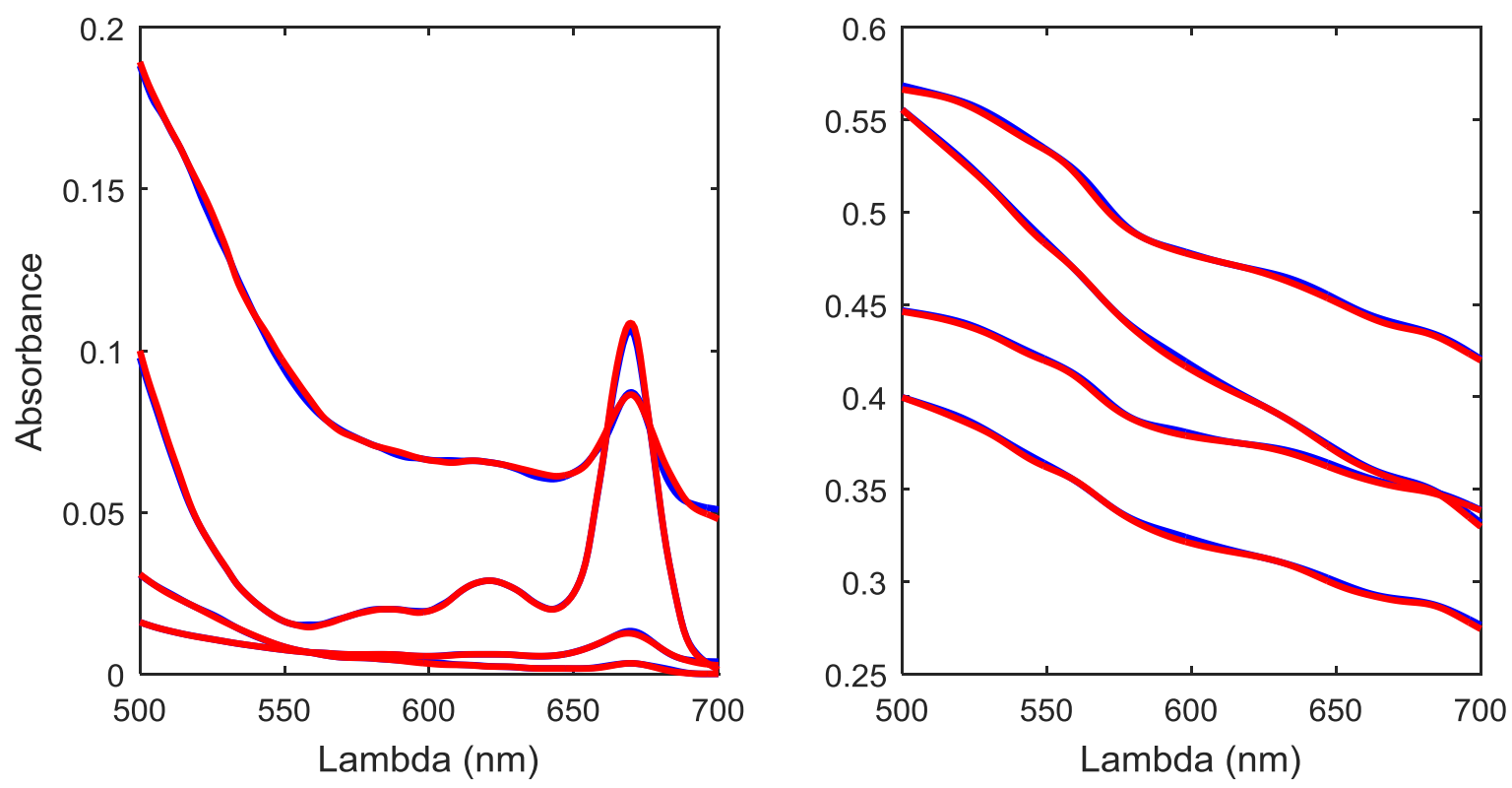

Supp Fig. 1. Four examples contrasting the measured (in blue) and estimated absorbance (in red) spectra for extracted pigments (left) and cultures (right) for Microcystis sp. PMC 816.12, 12.
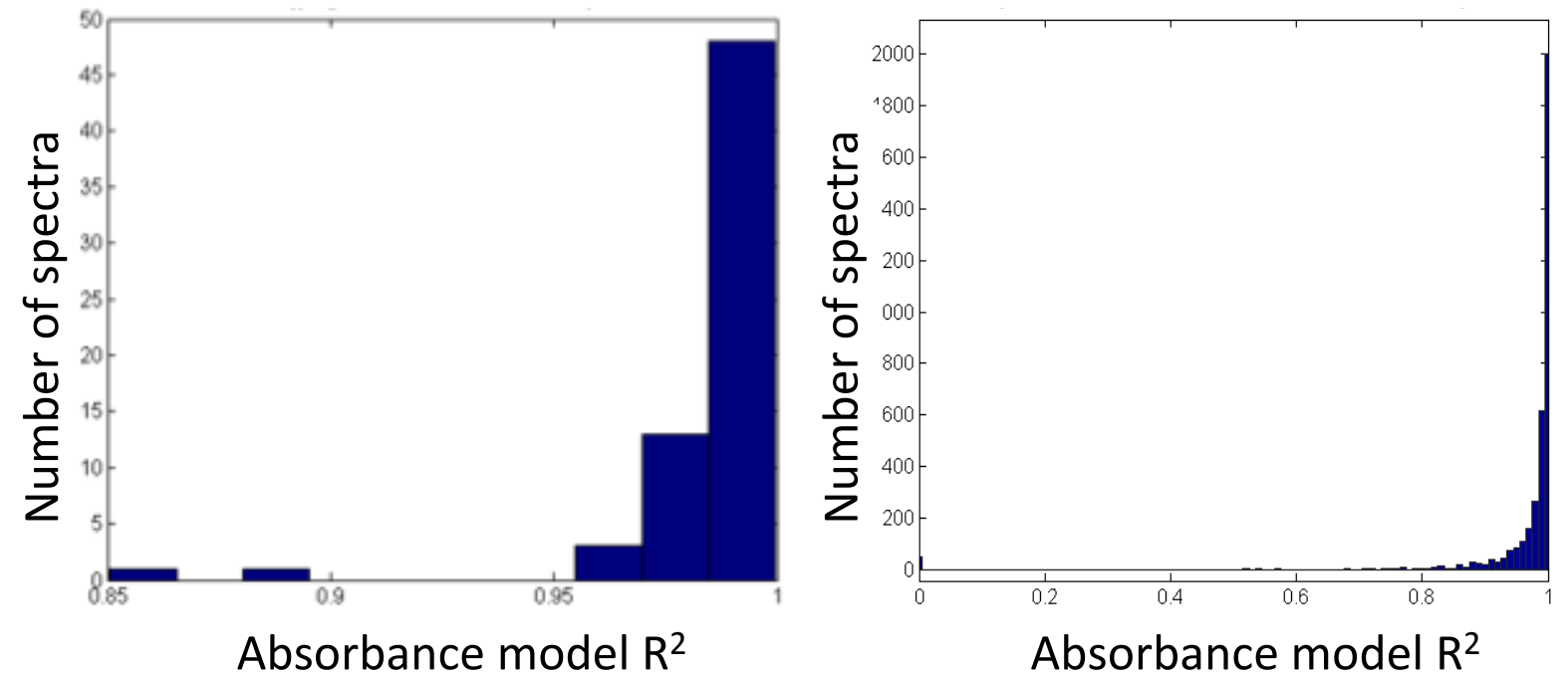

Supp Fig. 2. Distribution of the model's $\mathrm{R}^{2}$ for modeling the absorbance of extracted pigments (left) and the absorbance of monocultures (right) 